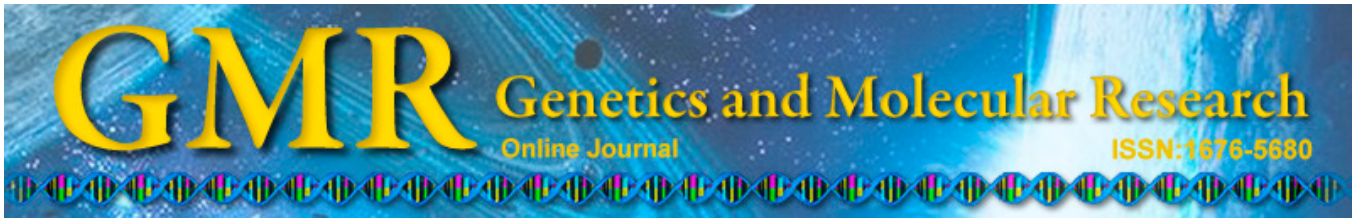

\title{
MTA1 promotes cell proliferation via DNA damage repair in epithelial ovarian cancer
}

\author{
Q.Y. Yang ${ }^{1}$, J.H. Li ${ }^{2}$, Q.Y. Wang ${ }^{3}$, Y. Wu ${ }^{3}$, J.L. Qin ${ }^{3}$, J.J. Cheng ${ }^{3}$ and J. Qiu ${ }^{3}$ \\ ${ }^{1}$ Department of Laboratory Medicine, \\ Tenth People's Hospital of Tongji University, Shanghai, China \\ ${ }^{2}$ Central Laboratory, \\ Tenth People's Hospital of Tongji University, Shanghai, China \\ ${ }^{3}$ Department of Gynecology and Obstetrics, \\ Tenth People's Hospital of Tongji University, Shanghai, China \\ Corresponding author: J. Qiu \\ E-mail: Laboratorypan@aliyun.com
}

Genet. Mol. Res. 13 (4): 10269-10278 (2014)

Received January 17, 2014

Accepted June 13, 2014

Published December 4, 2014

DOI http://dx.doi.org/10.4238/2014.December.4.21

\begin{abstract}
We examined whether metastasis-associated gene 1 (MTA1) promotes cell proliferation via DNA damage repair in ovarian cancer. MTA1 was successfully down-regulated using small interfering RNA in the epithelial ovarian cancer cell lines SKOV-3 and OVCAR-3. Cell growth was evaluated through MTT and colony formation assays. Fluorescence-activated cell sorting analysis was used to evaluate the distribution of cells in the cell cycle, and cytotoxicity assays were performed to study cell sensitivity to cisplatin. A neutral comet assay was used to measure levels of ionizing radiation-induced DNA damage in SKOV-3 cells, and Western blot analyses were carried out to examine the expression of key proteins involved in DNA damage repair pathways. MTA1 knockdown markedly inhibited cell growth and led to S phase cell cycle arrest. In addition, MTA1 depletion conferred sensitivity of ovarian cancer cells to cisplatin. Moreover, MTA1 depletion increased the level of ionizing radiation-induced DNA damage and caused irreparable damage, which was illustrated by a remarkable increase and
\end{abstract}


persistent existence of a comet tail as well as protein expression levels of $\gamma \mathrm{H} 2 \mathrm{AX}$, pRPA, and pChk1, all of which play critical roles in DNA repair. Thus, MTA1 promotes the proliferation of epithelial ovarian cancer cells by enhancing DNA repair.

Key words: DNA repair; Metastasis-associated gene 1;

Cell proliferation; Ovarian carcinoma

\section{INTRODUCTION}

Ovarian carcinoma is the most lethal gynecological malignancy worldwide and ranks as the fifth leading cause of cancer mortality among women in the United States. Approximately $80-90 \%$ of primary malignant ovarian tumors are epithelial carcinomas, accounting for approximately $6 \%$ of all female cancers. In 2012, 22,280 new cases were diagnosed and 15,500 cancer-related deaths were reported (Siegel et al., 2012). The overall 5-year survival rate for this disease is $15-40 \%$ (Faught, 2009). This poor prognosis is attributed to late detection and a relative lack of efficient treatments. Over the past 3 decades, there have been many advances in chemotherapy for epithelial ovarian cancer (EOC). Currently, platinum-based chemotherapy following surgical debulking of tumors is the standard treatment for advanced EOC. However, approximately $70 \%$ of patients with advanced stage EOC experience recurrence and chemotherapy resistance (Matsuo et al., 2010). Therefore, to improve chemotherapy efficacy, identification and evaluation of molecular targets that may be candidates for EOC therapy are urgently needed.

Metastasis-associated gene 1 (MTA1) was originally identified in a cDNA library screen of metastatic and non-metastatic adenocarcinoma cell lines from rat mammary glands (Toh et al., 1994). MTA1 is interesting in cancer biology because of its dual nature as a corepressor or coactivator, as well as its widespread overexpression in human cancers ( $\mathrm{Li}$ et al., 2012). MTA1 is thought to play an essential role in the progression of ovarian cancer because of its significant up-regulation in ovarian cancer tissues in advanced cancer stages and higher Féderation Internationale de Gynécologie et d'Obstétrique grading (Li et al., 2012). MTA1 overexpression in the OVCAR-3 ovarian cancer cell line increases the capability of anchorage-independent growth in soft agar colony formation assays. This is accompanied by marked up-regulation of the oncogenic cytokine chemokine ligand 1, as well as down-regulation of estrogen receptor beta, which acts as a tumor suppressor in ovarian cancer. These findings indicate that MTA1 directly and indirectly regulates the progression of ovarian cancer (Dannenmann et al., 2008; Toh and Nicolson, 2009).

In addition to its instrumental role in oncogenesis, it was recently reported that MTA1, which is an integral component of the nucleosome remodeling and deacetylase complex $(\mathrm{NuRD})$, plays a role in the DNA damage response. Specifically, MTA1 is involved in the ionizing radiation (IR)-induced DNA damage response and the ultraviolet-induced DNA damage checkpoint pathway (Li and Kumar, 2010; Lan et al., 2010). Moreover, MTA1 regulates DNA repair in a p53-dependent and -independent manner and facilitates the repair of DNA doublestrand breaks (DSBs) resulting from exposure to genotoxic stress (Li et al., 2010b).

Radiation and genotoxic drugs have been used clinically for years to fight cancer; however, efficient DNA repair in cancer cells contributes to chemo- and radio-resistance. 
Thus, targeting DNA repair mechanisms in cancer cells affords a therapeutic opportunity to selectively overcome this deficiency. Because MTA1 is involved in DNA damage repair and promotes cell growth, we hypothesized that MTA1 is a target for enhancing the therapeutic efficacy of IR and DNA-damaging drugs in ovarian cancer. In the current study, we examined whether MTA1 is a DNA repair protein that promotes the proliferation of ovarian cancer cells and assessed the sensitivity of cells depleted of MTA1 to cisplatin (CDDP) and IR. Down-regulation of MTA1 inhibited the proliferation of ovarian cancer cells, as well as caused cell cycle S phase arrest. Furthermore, MTA1 knockdown increased ovarian cancer cell sensitivity to CDDP and IR. In addition, MTA1 depletion resulted in the prolonged expression of phosphorylated $\mathrm{H} 2 \mathrm{AX}(\gamma \mathrm{H} 2 \mathrm{AX})$, phosphorylated replication protein A (pRPA), and phosphorylated checkpoint kinase 1 (pChk1), potential markers of irreparable DNA damage. In conclusion, MTA1 may be a promising molecular target for ovarian cancer therapy.

\section{MATERIAL AND METHODS}

\section{Cell culture and small interfering RNA (siRNA) transfection}

The human ovarian cancer cell lines SKOV-3 and OVCAR-3 were obtained from the American Type Culture Collection (ATCC, Manassas, VA, USA). All cells were cultured in RPMI 1640 medium (Gibco, Carlsbad, CA, USA) supplemented with $10 \%$ fetal bovine serum (HyClone, Waltham, MA, USA), $100 \mathrm{U} / \mathrm{mL}$ penicillin (Invitrogen), and $100 \mu \mathrm{g} / \mathrm{mL}$ streptomycin (Invitrogen) at $37^{\circ} \mathrm{C}$ and $5 \% \mathrm{CO}_{2}$. The siRNA against MTA1 (\#42272) and scrambled siRNA (\#4611), designed and synthesized by Sigma (St. Louis, MO, USA), were transiently transfected into SKOV-3 and OVCAR-3 cells using Lipofectamine 2000 (Invitrogen) according to manufacturer instructions.

\section{Cell growth and cell cytotoxicity assays}

Cell proliferation of EOC cell lines was measured using MTT assays. Briefly, cells transfected with si-MTA1-1/2 or negative control (NC) siRNA on 6-well plates were seeded onto 96-well plates after $8 \mathrm{~h}$. Next, $20 \mu \mathrm{L} 5 \mathrm{mg} / \mathrm{mL}$ MTT was added to the plates, followed by further incubation for $4 \mathrm{~h}$. Next, the medium was replaced with $150 \mu \mathrm{L}$ dimethyl sulfoxide (Amresco, Solon, OH, USA) and the absorbance was measured at $490 \mathrm{~nm}$ using a Model 550 Microplate Reader (Bio-Rad, Hercules, CA, USA). To assess the sensitivity of cells to chemotherapy following MTA1 knockdown, different concentrations of CDDP were added to each well $24 \mathrm{~h}$ after transfection. The cells were further incubated for $72 \mathrm{~h}$ before evaluating cell viability.

\section{Colony formation assay}

For colony formation assays, cells transfected with MTA1 or NC siRNA were seeded onto 12-well plates. After 7-10 days, colonies were fixed with formalin and stained with formaldehyde-crystal violet.

\section{Cell cycle analysis}

For cell cycle analysis, cells transfected with MTA1 or NC siRNA were fixed and 
stained with propidium iodide, followed by fluorescence-activated cell sorter analysis using a flow cytometer from BD Biosciences (Franklin Lakes, NJ, USA).

\section{Neutral comet assay}

Cells transfected with MTA1 siRNA were subjected to neutral comet assays to detect DNA damage using the comet assay system from Trevigen (Gaithersburg, MD, USA), according to manufacturer protocols. Comet tail moments were scored using the CASP software, version 1.2.3.

\section{Western blot analyses}

Cells were harvested and lysed in RIPA buffer [50 mM Tris, $\mathrm{pH} 8.0,150 \mathrm{mM} \mathrm{NaCl}$, $1 \%$ NP40, $0.5 \%$ doxycholine, $0.1 \%$ sodium dodecyl sulfate, $1 \mathrm{mM}$ phenylmethylsulfonyl fluoride, protease inhibitor cocktail tablet (Roche, Basel, Switzerland)]. A total of $40 \mu \mathrm{g}$ protein was resolved by sodium dodecyl sulfate-polyacrylamide gel electrophoresis, followed by electrophoretic transfer to polyvinylidene fluoride membranes (Millipore, Billerica, MA, USA). The membranes were blocked with 5\% nonfat milk in Tris-buffered saline, $\mathrm{pH} 7.4$, for $1 \mathrm{~h}$, and then incubated with the appropriate primary antibodies, followed by incubation with a horseradish peroxidase-conjugated secondary antibody (Zymed, San Francisco, CA, USA). Proteins were visualized by chemiluminescence and protein levels were determined by scanning gels and assessing band densities using the Image-J software (NIH, Bethesda, MD, USA).

\section{Statistical analysis}

Statistical analyses were conducted using the SPSS software, version 17.0 (SPSS, Chicago, IL, USA). Data are reported as means \pm standard deviation. Unless otherwise noted, statistical comparisons between experimental groups were analyzed by the Student paired $t$ test, and two-tailed $\mathrm{P}$ values less than 0.05 were considered to be statistically significant.

\section{RESULTS}

\section{MTA1 knockdown inhibits cell proliferation and causes $\mathbf{S}$ phase cell cycle arrest}

According to previous reports, $M T A 1$ is widely up-regulated in numerous human cancers (Dannenmann et al., 2008; Li et al., 2009). To determine the biological function of MTA1 in EOC, 2 types of synthesized siRNAs against MTA1 were transfected into SKOV-3 and OVCAR-3 cells. Western blot analysis showed that both siRNAs reduced MTA1 expression (Figure 1). Cell viability evaluated by MTT analyses revealed that knockdown of MTA1 significantly inhibited the growth of both EOC cell lines (Figure 2A and B), demonstrating the stimulatory role of MTA1 in ovarian cancer cell growth. Moreover, colony formation analyses consolidated the proliferation promoting effects of MTA1 (Figure 2C and D). We performed fluorescenceactivated cell sorting analysis to determine whether cell cycle changes occurred in MTA1depleted ovarian cancer cells. The results demonstrated that depletion of MTA1 caused notable $\mathrm{S}$ phase cell cycle arrest in SKOV-3 and OVCAR-3 cells (Figure 3A and B). Collectively, these results indicate that MTA1, as an oncogene, promotes ovarian cancer progression. 


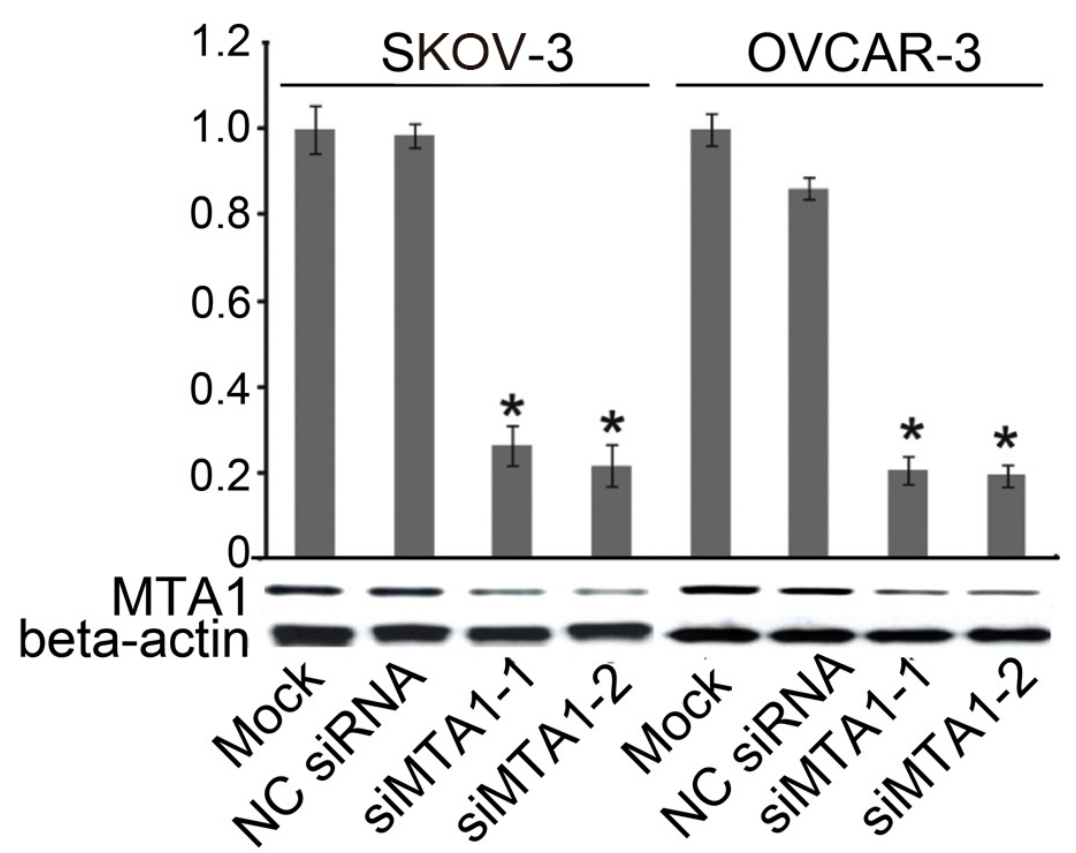

Figure 1. MTA1 is efficiently inhibited by siRNAs in SKOV-3 and OVCAR-3 cells.

A

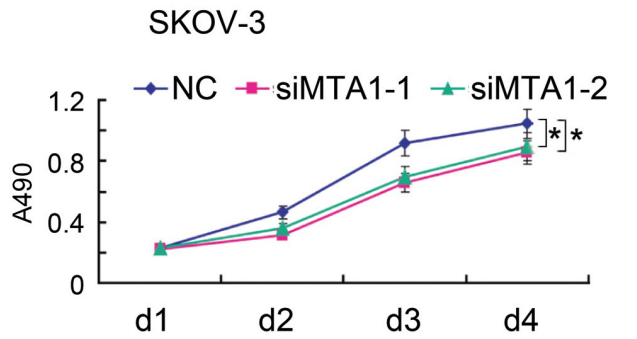

C

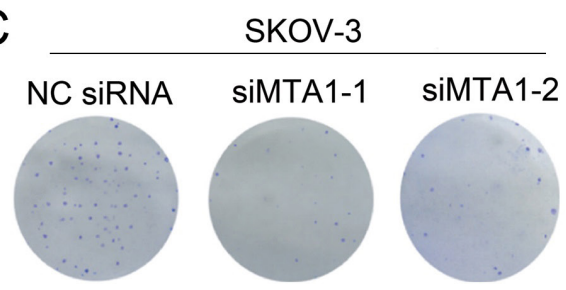

B

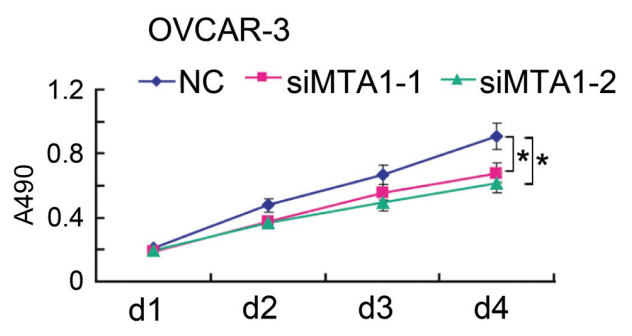

$\mathrm{D}$ OVCAR-3

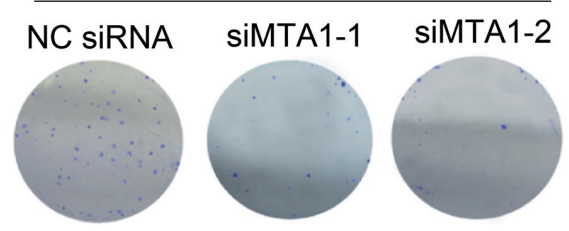

Figure 2. MTA1 knockdown prominently inhibits EOC cell proliferation. SKOV-3 (A) and OVCAR-3 (B) cells transfected with negative control (NC) siRNA or MTA1 siRNAs and subjected to MTT analysis at the indicating times. MTA1 knockdown led to significant inhibition of cell proliferation. Data are reported as means \pm standard deviation (SD) from three independent experiments. Colony formation assays performed to confirm the inhibitory function in SKOV-3 (C) and OVCAR-3 (D) cells upon depletion of MTA1. 


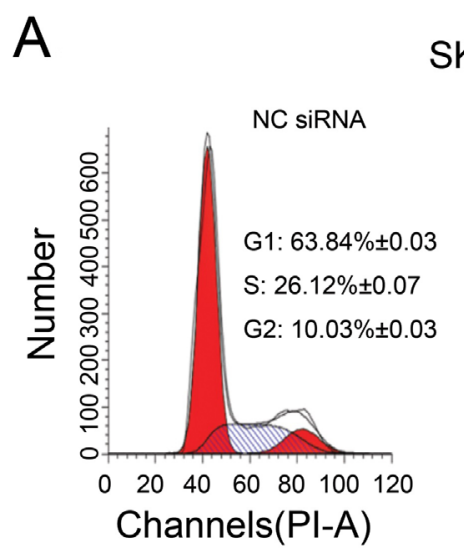

\section{SKOV-3}
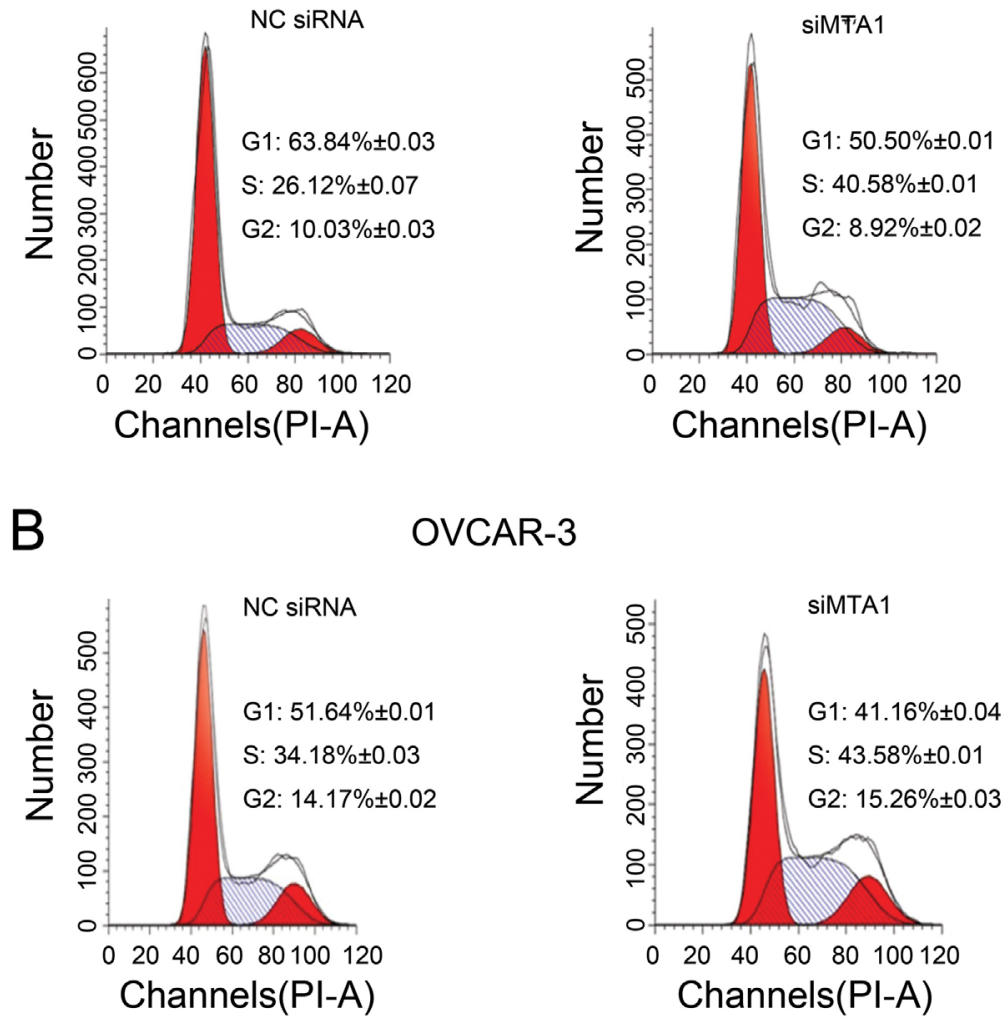

Figure 3. MTA1 deletion caused S phase cell cycle arrest. Fluorescence-activated cell sorting analysis performed to examine the distribution of cell cycle in MTA1-depleted SKOV-3 (A) and OVCAR-3 (B) cells. Data are reported as means $\pm \mathrm{SD}$ from at least three independent experiments.

\section{MTA1 is required for chemo- and radio-resistance in ovarian cancer cells}

Although it is known that MTA1 overexpression results in increased DNA DSB repair and decreased DNA damage sensitivity following IR treatment in p53-null cells (Li et al., 2010b), no studies have explored the role of MTA1 in the DNA damage response of ovarian cancer cells. Therefore, because platinum-based chemotherapy following surgical tumor debulking is the standard treatment for advanced EOC, and IR is ubiquitously used in the therapy of many cancers, we investigated the possible therapeutic role of MTA1 in ovarian cancer cells following CDDP and IR treatment. As shown in Figure 4A, MTA1 knockdown reduced cell viability following CDDP treatment, demonstrating increased sensitivity to CDDP. The half maximal inhibitory concentration values further confirmed that MTA1 deletion reduced cell resistance to CDDP (Figure 4B). Next, we evaluated cell sensitivity to IR by performing a neutral comet assay, a classic and efficient method for detecting DNA damage (Cortés-Gutiérrez et al., 2012) that is represented by a specific parameter known as the comet tail moment. This 
value is calculated as the product of DNA tail length and the intensity of DNA in the tail. Ovarian cancer cells transiently transfected with MTA1 or NC siRNA were exposed to 20 Gy IR, followed by detection of DNA at the indicated time. As shown in Figure 4C and D, cells depleted of MTA1 exhibited severe DNA damage compared to control cells. Interestingly, in the absence of IR treatment, knockdown of MTA1 also caused endogenous DNA damage, and MTA1-depleted cells did not show significantly more DNA damage immediately after IR exposure. However, over time, MTA1-depleted cells displayed prolonged damage compared to control cells. Together, these data demonstrate a role for MTA1 in DNA repair, suggesting that MTA1 is a therapeutic target for reducing chemo- and radio-resistance in ovarian cancers.

A

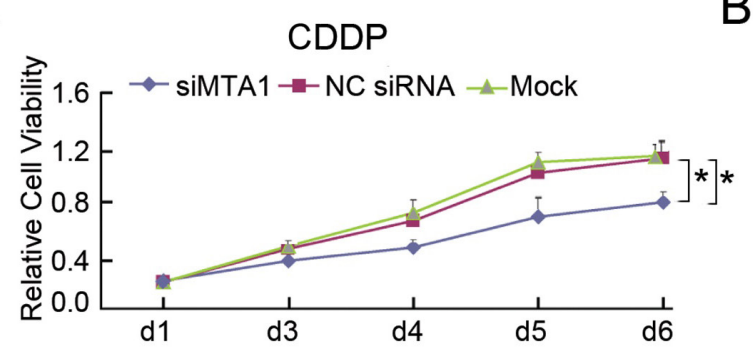

C

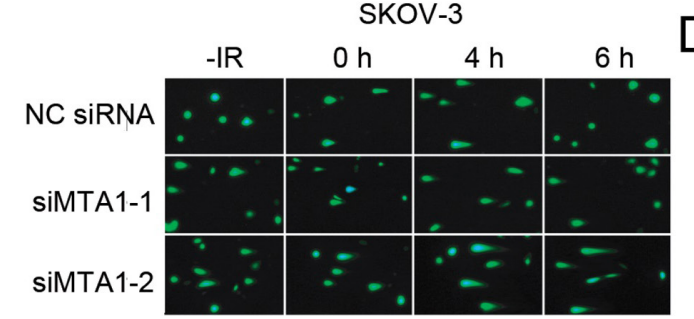

B
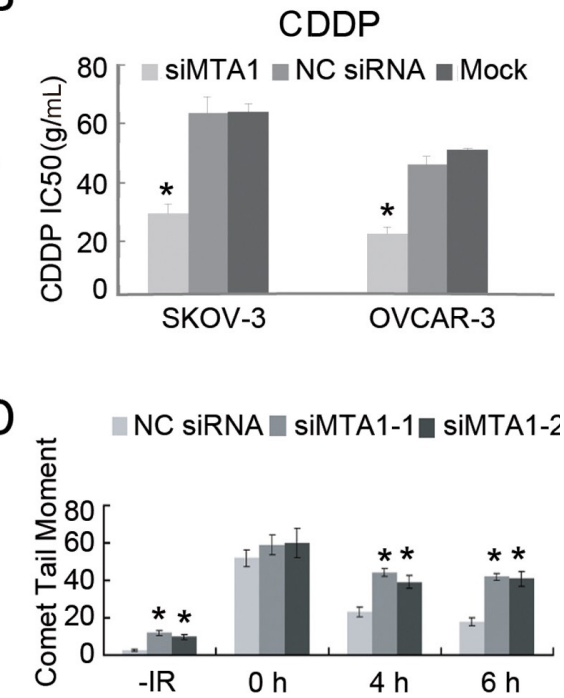

Figure 4. MTA1 is required for chemo- and radio-resistance in ovarian cancer cells. A. SKOV-3 cells transfected with NC or MTA1 siRNAs and treated with CDDP for $12 \mathrm{~h}$, followed by further incubation with fresh medium for the indicating times before the MTT assay. B. IC $_{50}$ values of SKOV-3 cells transfected with NC or MTA1 siRNAs assessed using SPSS. Data are reported as means \pm SD from three independent experiments, $* \mathrm{P}<0.05$. C. Fortyeight hours after transfection with NC or MTA1 siRNAs, SKOV-3 cells were untreated or irradiated with 20 Gy IR, and harvested at the indicated times for neutral comet assays. D. Migration length of DNA fragments analyzed by the CASP_1.2.3 software from 100 cells for each time point, demonstrated as the comet tail moment. Data are reported as means $\pm \mathrm{SD}$ from three independent experiments, ${ }^{*} \mathrm{P}<0.05$.

\section{Impact of MTA1 on several DNA damage response proteins}

During DNA replication, the progress of the replication fork over DNA breaks is stalled in a cell until damage can be repaired or bypassed. Chk1 and RPA are the predominant regulators of the cellular response induced by stalled replication forks in the intra-S phase, a response that leads to the inhibition of DNA replication initiation at origins of replication. Therefore, we investigated the regulatory functions of Chk1 and RPA in MTA1 downregulated cells following IR treatment. First, we detected the widely recognized damage biomarker $\gamma \mathrm{H} 2 \mathrm{AX}$ via Western blot analysis. The band intensities revealed that knockdown 
of MTA1 led to higher expression of $\gamma \mathrm{H} 2 \mathrm{AX}$ in ovarian cancer cells immediately after IR treatment. Additionally, $\gamma \mathrm{H} 2 \mathrm{AX}$ was continuously expressed in MTA1-deleted cells, while it was reduced $6 \mathrm{~h}$ after IR treatment in control cells (Figure 5A and B). Thus, consistent with data from the comet assays, MTA1 plays a pronounced role in mediating resistance to DNA damage. Next, to explore the involvement of DNA damage response proteins, Western blot analysis was performed to examine the protein levels of total and phosphorylated Chk1 and RPA. As illustrated in Figure 3A and B, MTA1 knockdown led to increased expression of both Chk1 phosphorylated at Ser317 (pChk1) and pRPA, without affecting total expression levels. Furthermore, expression of pChk1 and pRPA significantly decreased $6 \mathrm{~h}$ after IR in control cells, but remained high in MTA1-depleted cells, suggesting irreparable DNA damage in these cells.
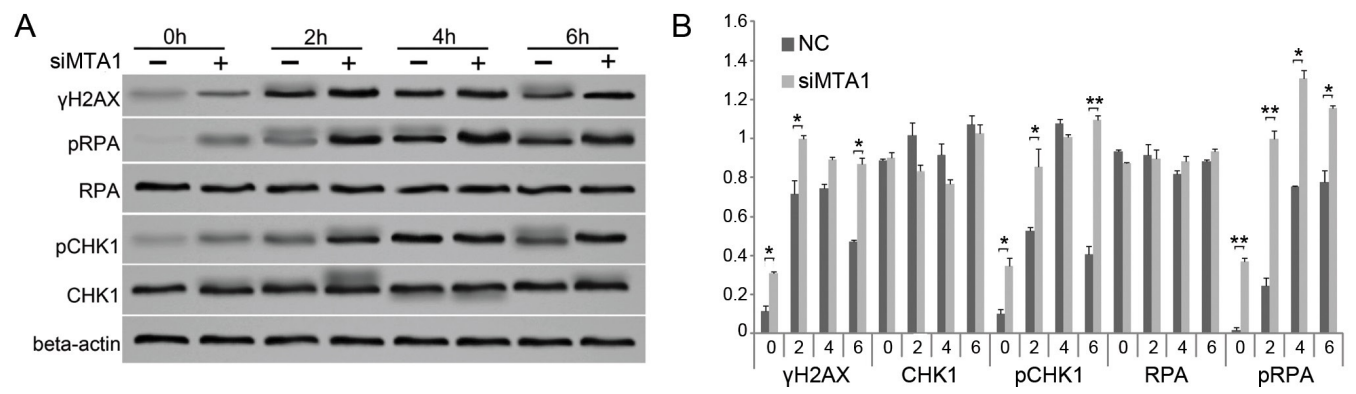

Figure 5. Effect of MTA1 on DNA damage response proteins. A. Cell lysates from SKOV-3 cells treated with 20 Gy IR after transfection with NC or MTA1 siRNAs for $48 \mathrm{~h}$, and subjected to Western blot analysis using antibodies against $\gamma \mathrm{H} 2 \mathrm{AX}, \mathrm{RPA}, \mathrm{pRPA}, \mathrm{Chk} 1$, and $\mathrm{pChk} 1$ at the indicated time points. B. The gray scale values from three independent Western blots scanned using Image-J software, and the relative expression levels of these proteins were determined using the Student paired $t$-test, $* \mathrm{P}<0.05$.

\section{DISCUSSION}

Most ovarian-cancer-related deaths are attributed to tumor metastasis and chemo-resistance. The development of molecular-targeted therapeutic strategies is necessary for overcoming the low efficacy of current platinum-based standard chemotherapy in ovarian cancer. MTA1 has gained widespread attention as a therapeutic target because it is up-regulation is distinctly correlated with the malignant properties of a wide variety of cancers, including ovarian cancer (Yi et al., 2003; Dannenmann et al., 2008). Dannenmann et al. (2008) showed that cell clones expressing MTA1 rapidly grew to multicellular tumor spheroids, whereas control cells remained mostly singular. Mazumdar et al. (2001) demonstrated that MTA1 overexpression in the MCF-7 breast cancer cell line was accompanied by enhancement of the ability of cells to grow in an anchorage-independent manner. Furthermore, an in vivo study by Bagheri-Yarmand et al. (2004) showed that overexpression of the mammary tumor-like virus-MTA1 transgene in mouse mammary glands resulted in increased cell proliferation. Consistent with these reports, in our study, we used an siRNA-based approach to knockdown MTA1, which led to marked inhibition of ovarian cancer cell proliferation. In conjugation with this inhibitory function, MTA1 evidently caused S phase arrest, indicating a possible role of MTA1 in oncogenesis.

MTA1 has recently been recognized as an effective DNA damage response protein, linking 2 previously unconnected NuRD complex and DNA damage response pathways (Li 
et al., 2010b). Li et al. (2010b) suggested an inherent role of the MTA1-p53-p53R2 pathway in the DNA damage response in cancer cells. In addition, the presence of an additional p53independent role of MTA1 in the DNA damage response, that acts in part by modulating the p21(WAF1)-PCNA pathway, has also been identified. Thus, we explored the underlying mechanism of MTA1 in promoting ovarian cancer cell growth with respect to the DNA damage response. Depletion of MTA1 made ovarian cancer cells more sensitive to CDDP and IR treatment in vitro. Furthermore, knockdown of MTA1 resulted in persistent DNA damage in response to $20 \mathrm{~Gy} \mathrm{IR,} \mathrm{demonstrating} \mathrm{the} \mathrm{irreparable} \mathrm{damage} \mathrm{in} \mathrm{these} \mathrm{cells.} \mathrm{At}$ the molecular level, we also detected a pronounced increase in the expression of $\gamma \mathrm{H} 2 \mathrm{AX}$, a widely accepted damage biomarker. Our data demonstrate that MTA1 promotes cell growth by mediating double-strand break repair. In accordance with our results, other studies reported that MTA1 depletion results in increased DNA damage sensitivity and decreased DSB repair after exposure to IR (Zhu et al., 2009).

Recently, Li et al. (2010a) reported that depletion of MTA1 compromised ATR-mediated Chk1 activation following UV treatment, accompanied by the salient down-regulation of Chk1 and its interacting partner claspin. Niziolek-Kierecka et al. (2012) showed that prolonged Chk1 phosphorylation may serve as a potential marker for persistent DNA damage in a study of polycyclic aromatic hydrocarbons-induced damage in HepG2 cells. Moreover, RPA as a DNA damage sensor is involved in the initial recognition of damaged DNA or stalled replication forks, which is followed by phosphorylation regulated by Chk1 during the $\mathrm{S}$ phase checkpoint pathway in vitro (Liu et al., 2006). Thus, $\gamma \mathrm{H} 2 \mathrm{AX}$, pChk1, and pRPA may serve as biomarkers of DNA damage; in our study, the prolonged expression of these proteins in MTA1-deleted cells resulted in impaired damage repair.

Increased chemo-resistance and radio-resistance of cancer cells is a major obstacle in the treatment and management of malignant cancers (Zhu et al., 2009). An important mechanism underlying the development of such therapeutic resistance is that cancer cells recognize DNA lesions induced by DNA-damaging agents or by IR, and repair these lesions by activating various DNA repair pathways (Kelley and Fishel, 2008). Therefore, several anticancer strategies targeting the DNA damage response have been proposed, some of which are currently being tested in preclinical and clinical trials. One of the ATM inhibitors (KU55933) has been shown to effectively sensitize cancer cells to DNA-damaging drugs (Hickson et al., 2004). Downstream of ATM, inhibitors of Chk1 and Chk2 protein kinases have also been proposed to enhance the cytotoxicity of antitumor drugs (O'Connor et al., 2007). Our data demonstrate that MTA1 mediates DNA repair, and thus may be a therapeutic candidate in cancer, particularly ovarian cancer. However, for the clinical application, additional studies are necessary.

\section{Conflicts of interest}

The authors declare no conflict of interest.

\section{REFERENCES}

\footnotetext{
Bagheri-Yarmand R, Talukder AH, Wang RA, Vadlamudi RK, et al. (2004). Metastasis-associated protein 1 deregulation causes inappropriate mammary gland development and tumorigenesis. Development 131: 3469-3479.

Cortés-Gutiérrez EI, Hernández-Garza F, García-Perez JO, Dávila-Rodríguez MI, et al. (2012). Evaluation of DNA single and double strand breaks in women with cervical neoplasia based on alkaline and neutral comet assay techniques. $J$. Biomed. Biotechnol. 2012: 385245.
} 
Dannenmann C, Shabani N, Friese K, Jeschke U, et al. (2008). The metastasis-associated gene MTA1 is upregulated in advanced ovarian cancer, represses ERbeta, and enhances expression of oncogenic cytokine GRO. Cancer Biol. Ther. 7: 1460-1467.

Faught W (2009). Cancer of the ovary: there is reason for optimism. J. Obstet. Gynaecol. Can 31: 297-302.

Hickson I, Zhao Y, Richardson CJ, Green SJ, et al. (2004). Identification and characterization of a novel and specific inhibitor of the ataxia-telangiectasia mutated kinase ATM. Cancer Res. 64: 9152-9159.

Kelley MR and Fishel ML (2008). DNA repair proteins as molecular targets for cancer therapeutics. Anticancer Agents Med. Chem. 8: 417-425.

Lan L, Ui A, Nakajima S, Hatakeyama K, et al. (2010). The ACF1 complex is required for DNA double-strand break repair in human cells. Mol. Cell 40: 976-987.

Li DQ and Kumar R (2010). Mi-2/NuRD complex making inroads into DNA-damage response pathway. Cell Cycle 9: 2071-2079.

Li DQ, Ohshiro K, Reddy SD, Pakala SB, et al. (2009). E3 ubiquitin ligase COP1 regulates the stability and functions of MTA1. Proc. Natl. Acad. Sci. U. S. A. 106: 17493-17498.

Li DQ, Pakala SB, Reddy SD, Ohshiro K, et al. (2010a). Revelation of p53-independent function of MTA1 in DNA damage response via modulation of the p21 WAF1-proliferating cell nuclear antigen pathway. J. Biol. Chem. 285: 10044-10052.

Li DQ, Ohshiro K, Khan MN and Kumar R (2010b). Requirement of MTA1 in ATR-mediated DNA damage checkpoint function. J. Biol. Chem. 285: 19802-19812.

Li DQ, Pakala SB, Nair SS, Eswaran J, et al. (2012). Metastasis-associated protein 1/nucleosome remodeling and histone deacetylase complex in cancer. Cancer Res. 72: 387-394.

Liu JS, Kuo SR and Melendy T (2006). DNA damage-induced RPA focalization is independent of gamma-H2AX and RPA hyper-phosphorylation. J. Cell Biochem. 99: 1452-1462.

Matsuo K, Eno ML, Im DD, Rosenshein NB, et al. (2010). Clinical relevance of extent of extreme drug resistance in epithelial ovarian carcinoma. Gynecol. Oncol. 116: 61-65.

Mazumdar A, Wang RA, Mishra SK, Adam L, et al. (2001). Transcriptional repression of oestrogen receptor by metastasisassociated protein 1 corepressor. Nat. Cell Biol. 3: 30-37.

Niziolek-Kierecka M, Dreij K, Lundstedt S and Stenius U (2012). GammaH2AX, pChk1, and Wip1 as potential markers of persistent DNA damage derived from dibenzo[a,1]pyrene and PAH-containing extracts from contaminated soils. Chem. Res. Toxicol. 25: 862-872.

O'Connor MJ, Martin NM and Smith GC (2007). Targeted cancer therapies based on the inhibition of DNA strand break repair. Oncogene 26: 7816-7824.

Siegel R, Naishadham D and Jemal A (2012). Cancer statistics, 2012. CA Cancer J. Clin. 62: 10-29.

Toh Y and Nicolson GL (2009). The role of the MTA family and their encoded proteins in human cancers: molecular functions and clinical implications. Clin. Exp. Metastasis 26: 215-227.

Toh Y, Pencil SD and Nicolson GL (1994). A novel candidate metastasis-associated gene, mta1, differentially expressed in highly metastatic mammary adenocarcinoma cell lines. cDNA cloning, expression, and protein analyses. J. Biol. Chem. 269: 22958-22963.

Yi S, Guangqi H and Guoli H (2003). The association of the expression of MTA1, nm23H1 with the invasion, metastasis of ovarian carcinoma. Chin. Med. Sci. J. 18: 87-92.

Zhu Y, Hu J, Hu Y and Liu W (2009). Targeting DNA repair pathways: a novel approach to reduce cancer therapeutic resistance. Cancer Treat. Ver. 35: 590-596. 